PROCEEDINGS OF THE

AMERICAN MATHEMATICAL SOCIETY

Volume 133, Number 3, Pages 859-863

S 0002-9939(04)07596-3

Article electronically published on September 29, 2004

\title{
THERE EXIST NO GAPS BETWEEN GEVREY DIFFERENTIABLE AND NOWHERE GEVREY DIFFERENTIABLE
}

\author{
SOON-YEONG CHUNG AND JAEYOUNG CHUNG \\ (Communicated by Carmen C. Chicone)
}

\begin{abstract}
We verify that there exist no gaps between Gevrey differentiable and nowhere Gevrey differentiable in the sense that for given $s>1$, there is a nowhere Gevrey differentiable function on $\mathbb{R}$ of order $s$ that is Gevrey differentiable of order $r$ for any $r>s$, which also gives a strong example that is Gevrey differentiable but nowhere analytic.
\end{abstract}

In the elementary course of analysis there are many examples of continuous functions that are nowhere differentiable.

Likewise, there are many concrete examples of smooth functions that are nowhere analytic (e.g., see [C] and [KK]). In particular, in [KK] an example was given of a smooth function that is increasing and nowhere analytic.

Lately analysts, especially those who are studying partial differential equations, are in need of classifying the smoothness of functions according to how close they are to analytic functions. To do so, Gevrey differentiability has been widely used to classify intermediate regularities between differentiability and analyticity.

For a real number $s>0$ and an open subset $\Omega$ of $\mathbb{R}$ an infinitely differentiable function $\phi$ in $\Omega$ is said to be Gevrey differentiable at $x_{0} \in \Omega$ of order $s$ if there exists a compact neighborhood $K$ of $x_{0}$ such that

$$
\sup _{x \in K}\left|\phi^{(p)}(x)\right| \leq C h^{p} p !^{s}, \quad p \in \mathbb{N}_{0}
$$

for some constants $C>0$ and $h>0$, where $\mathbb{N}_{0}$ denotes the set of nonnegative integers. We denote by $G^{s}(\Omega)$ the set of all functions that are Gevrey differentiable at each point $x_{0} \in \Omega$.

In particular, if $s=1$ the inequality (1) is just the Cauchy estimate for analytic functions. If $s<1$, then $G^{s}(\mathbb{R})$ consists of all analytic functions that extend to entire functions in the whole complex plane.

The smoothness of functions is usually divided according to the Gevrey order $s$, since the space $G^{s}(\Omega)$ becomes closer to the class $\mathcal{A}(\Omega)$ of (real) analytic functions in $\Omega$, as the Gevrey order $s$ decreases to 1 . Then, we have the following inclusion:

$$
\mathcal{A}(\Omega)=G^{1}(\Omega) \subset G^{s}(\Omega) \subset G^{r}(\Omega)
$$

for every $1 \leq s \leq r$.

Received by the editors November 13, 2003 and, in revised form, November 23, 2003.

2000 Mathematics Subject Classification. Primary 26A27, 26A99.

(C)2004 American Mathematical Society 
Note that the function

$$
f(t)= \begin{cases}\exp \left(-\frac{1}{t}\right), & t>0 \\ 0, & t \leq 0\end{cases}
$$

is widely known in advanced calculus as a simple example of a function that is infinitely differentiable but not real analytic on $\mathbb{R}$. As we shall see later, the above function belongs to $G^{2}(\mathbb{R})$.

In this note, for given $s>1$, we construct a function on $\mathbb{R}$ that belongs to $\bigcap_{r>s} G^{r}(\mathbb{R})$ but is nowhere Gevrey differentiable of order $s$. As a consequence, we also construct an increasing function that is Gevrey differentiable but nowhere analytic.

The following lemma gives a crucial example, which will be very useful later.

Lemma 1. For $s>1$ let $f_{s}$ denote the function on $\mathbb{R}$ defined by

$$
f_{s}(t)= \begin{cases}\exp \left(-t^{-\frac{1}{s-1}}\right), & t>0 \\ 0, & t \leq 0\end{cases}
$$

Then $f_{s}$ belongs to $G^{s}(\mathbb{R})$.

Proof. Using the Cauchy integral formula, for each $t>0$ we can write

$$
f_{s}^{(p)}(t)=\frac{p !}{2 \pi i} \int_{|z-t|=k t} \frac{\exp \left(-z^{-\frac{1}{s-1}}\right)}{(z-t)^{p+1}} d z
$$

for all $0<k<1$. Thus it follows that

$$
\left|f_{s}^{(p)}(t)\right| \leq p !|k t|^{-p} \sup _{|z-t|=k t}\left|\exp \left(-z^{-\frac{1}{s-1}}\right)\right|
$$

for all $0<k<1$.

Let $k_{0}=\sin \theta_{0}, \theta_{0}=\min \left\{\frac{\pi}{3}, \frac{\pi}{3}(s-1)\right\}$. Then for every $t>0$,

$$
\left|f_{s}^{(p)}(t)\right| \leq p !\left|k_{0} t\right|^{-p} \exp \left(-\left(1+k_{0}\right)^{-\frac{1}{s-1}} \cos \left(\theta_{0} /(s-1)\right) t^{-\frac{1}{s-1}}\right) .
$$

The right-hand side of (2) tends to zero as $t \rightarrow 0^{+}$, so that $f_{s}(t)$ is infinitely differentiable in $\mathbb{R}$. Moreover, if $L>0$, then we have by Stirling's formula,

$$
\sup _{0<t<\infty} t^{-p} \exp \left[-L t^{-\frac{1}{s-1}}\right]=\left(\frac{s-1}{L e}\right)^{(s-1) p} p^{(s-1) p} \leq\left(\frac{s-1}{L}\right)^{(s-1) p} p !^{s-1} .
$$

Thus if we take the supremum for $t>0$ in the right-hand side of (2) we have

$$
\sup _{t \in \mathbb{R}}\left|f_{s}^{(p)}(t)\right| \leq H^{p} p !^{s}, p \in \mathbb{N}_{0},
$$

where

$$
H=\left(\frac{1+\sin \theta_{0}}{\sin \theta_{0}}\right)\left(\frac{s-1}{\cos \left(\theta_{0} /(s-1)\right)}\right)^{s-1} .
$$

This completes the proof.

It is well known that the space $G^{s}(\mathbb{R})$ forms a ring with respect to the arithmetic product of functions and is stable under analytic change of coordinates (see Propositions 1.4.5 and 1.4.6 in $[\mathbf{R}]$ ). Thus the function $\psi_{s}$ defined by

$$
\psi_{s}(x)=f_{s}(x) f_{s}(1-x), x \in \mathbb{R},
$$


is still Gevrey differentiable of order $s>1$ and (real) analytic in the interval $(0,1)$, since $f_{s}$ is analytic for $x>0$. Moreover, the support of $\psi_{s}$ is exactly the interval $[0,1]$ and $\psi_{s}^{(p)}(0)=\psi_{s}^{(p)}(1)=0$ for all $p \in \mathbb{N}_{0}$.

Now we are able to construct a desired function.

Theorem 2. For $s>1$ there exists a function $\Psi_{s}$ that is nowhere Gevrey differentiable in $\mathbb{R}$ of order $s$ but Gevrey differentiable of order $r$ for all $r>s$.

Proof. Using the function $\psi_{s}$ in (4) we define

$$
\Psi_{s}(x)=\sum_{n=0}^{\infty} e^{-n !} \psi_{s}\left(2^{n} x-\left[2^{n} x\right]\right),
$$

where $[x]$ denotes the greatest integer function. First we show that $\Psi_{s}$ is Gevrey differentiable of arbitrary order $r>s$. In view of (3) and (4) there exist constants $C>0$ and $h>0$ such that

$$
\sup _{x \in \mathbb{R}}\left|\psi_{s}^{(p)}(x)\right| \leq C h^{p} p !^{s}, \quad p \in \mathbb{N}_{0} .
$$

Thus each derivative of partial sums of the right-hand side of (5) converges uniformly. It follows that $\Psi_{s}$ is infinitely differentiable in $\mathbb{R}$ and that for every $x \in \mathbb{R}$ and for every $p \in \mathbb{N}_{0}$,

$$
\begin{aligned}
\left|\Psi_{s}^{(p)}(x)\right| & \leq \sum_{n=0}^{\infty} e^{-n !} 2^{n p}\left|\psi_{s}^{(p)}\left(2^{n} x-\left[2^{n} x\right]\right)\right| \\
& \leq C h^{p} p !^{s} \sum_{n=0}^{\infty} e^{-n !} 2^{n p} \\
& \leq C^{\prime} h^{p} p !^{s} \sup _{n \in \mathbb{N}_{0}} e^{-n !+n} 2^{n p} .
\end{aligned}
$$

Now it is easy to see that for every $\delta>0$ there exists $C_{\delta}>0$ such that

$$
e^{-n !+n} \leq C_{\delta} e^{-\delta 2^{n / \delta}} .
$$

Using the inequality

$$
e^{-\delta x} \leq\left(\frac{p !}{x^{p}}\right)^{\delta}, p \in \mathbb{N}_{0}, x>0,
$$

we have

$$
\sup _{n \in \mathbb{N}_{0}} e^{-\delta 2^{n / \delta}} 2^{n p} \leq p !^{\delta} .
$$

It follows that for every $\delta>0$ there exists $C_{\delta}$ such that

$$
\left|\Psi_{s}^{(p)}(x)\right| \leq C^{\prime} C_{\delta} h^{p} p !^{s+\delta},
$$

which implies that $\Psi_{s}$ is Gevrey differentiable of order $r$ for each $r>s$.

Now it remains to show that $\Psi_{s}$ is nowhere Gevrey differentiable of order $s$. Let $Q$ be the set of all points of the form $2^{-m} k, m \in \mathbb{N}_{0}, k \in \mathbb{Z}$, where $\mathbb{Z}$ denotes the set of integers. Then $Q$ is a dense subset of $\mathbb{R}$. Thus it suffices to show that $\Psi_{s}$ is not Gevrey differentiable of order $s$ at each point in $Q$. On the contrary, we suppose that $\Psi_{s}$ is Gevrey differentiable of order $s$ at some point $x_{0} \in Q$. Let $x_{0}=2^{-m_{0}} k_{0}$. Here we may assume that $2^{m_{0}}$ and $k_{0}$ are relatively prime. Then, for 
$n=0,1,2, \ldots, m_{0}-1$, each function $\psi_{s}\left(2^{n} x-\left[2^{n} x\right]\right)$ is analytic at $x_{0}$ and hence Gevrey differentiable of order $s$ at $x_{0}$, and the remainder term

$$
\Theta_{m_{0}}(x):=\sum_{n=m_{0}}^{\infty} e^{-n !} \psi_{s}\left(2^{n} x-\left[2^{n} x\right]\right)
$$

is Gevrey differentiable of order $s$ at $x_{0}$. Now we may assume that $x_{0}=0$ since $\Theta_{m_{0}}(x)$ is a periodic function of period $2^{-m_{0}}$. Then there exist constants $C>0$ and $h>0$ such that

$$
\sup _{|t| \leq \epsilon}\left|\Theta_{m_{0}}^{(p)}(t)\right| \leq C h^{p} p !^{s}, p \in \mathbb{N}_{0},
$$

for some small $\epsilon$. Since, for $x>0$,

$$
\Theta_{m_{0}}(x)=\int_{0}^{x} \frac{(x-t)^{p-1}}{(p-1) !} \Theta_{m_{0}}^{(p)}(t) d t
$$

we have

$$
0 \leq \Theta_{m_{0}}(x) \leq C h^{p} x^{p} p !^{s-1}, p \in \mathbb{N}_{0},
$$

for every $x$ with $0<x \leq \epsilon$. Thus it follows that

$$
0 \leq e^{-n !} \psi_{s}\left(2^{n} x-\left[2^{n} x\right]\right) \leq C h^{p} x^{p} p !^{s-1}, p \in \mathbb{N}_{0},
$$

for every $n \geq m_{0}$ and $0<x \leq \epsilon$.

While if we put $x=2^{-n} p^{-(s-1)}$ with $p$ sufficiently large,

$$
e^{-n !} \psi_{s}\left(p^{-(s-1)}\right) \leq C\left(2^{-n} h\right)^{p}\left(p^{-p} p !\right)^{s-1},
$$

from which we have

$$
e^{p} \psi_{s}\left(p^{-(s-1)}\right) \leq C e^{n !}\left(2^{-n} e h\right)^{p}\left(p^{-p} p !\right)^{s-1}
$$

for all $n \geq m_{0}$ and all large $p$. Then the left-hand side of (6) goes to 1 as $p \rightarrow \infty$, but if we choose $n \geq m_{0}$ and $2^{-n} e h<1$, the right-hand side goes to 0 as $p \rightarrow \infty$, which leads to a contradiction.

Corollary 3. For an arbitrary number $s>1$ there exists an increasing function that is Gevrey differentiable of arbitrary order $r>s$ but nowhere Gevrey differentiable of order $s$.

Proof. Let $\Psi_{s}(x)$ be the function defined in (5). Then the function $\int_{0}^{x} \Psi_{s}(y) d y$ is the required one, since $\Psi_{s}(x)$ is positive except at a countable number of points.

As a direct consequence of the above result we have the following:

Corollary 4. For an arbitrary number $r>1$ there exists an increasing function that is Gevrey differentiable of order $r$ but nowhere analytic.

Remark. Finally we suggest two questions. Let $0<s \leq 1$ be given.

(1) Does there exist a nowhere Gevrey differentiable function of order $s$ that is Gevrey differentiable of order $r$ for every $r>s$ ?

(2) Does there exist a Gevrey differentiable function of order $s$ that is nowhere Gevrey differentiable of order $r$ for every $r<s$ ? 


\section{ACKNOWLEDGEMENT}

We would like to express our gratitude to the referee for useful comments and corrections of our paper. The first author was supported by Grant R01-1999-00000001-0 from KOSEF, and the second author was supported by Korean Research Foundation Grant KRF-2001-015-DP0011.

\section{REFERENCES}

[C] F. S. Cater, Differentiable, nowhere analytic functions, Amer. Math. Monthly 91 (1984), 618-624. MR86b:26034

[KK] S. S. Kim and K.H. Kwon, Smooth $\left(C^{\infty}\right)$ but nowhere analytic functions, Amer. Math. Monthly 107 (2000), 264-266.

[R] L. Rodino, Linear Partial Differential Operators in Gevrey Spaces, World Scientific, London, 1993. MF 95c:35001

Department of Mathematics, Sogang University, Seoul 121-742, Korea

E-mail address: sychung@ccs.sogang.ac.kr

Department of Mathematics, Kunsan National University, Kunsan 573-701, Korea

E-mail address: jychung@kunsan.ac.kr 